Review Article

\title{
Dual Contraceptive Use and Factor Associated with People Living with HIV/AIDS: A Systematic Review and Meta-Analysis
}

\author{
Yibeltal Mesfin $\mathbb{D}^{1},{ }^{1}$ Muche Argaw $\mathbb{D}^{\mathbb{D}},{ }^{1}$ Shegaw Geze $\mathbb{D}^{1},{ }^{1}$ and Bitew Tefera $\mathbb{D}^{2}$ \\ ${ }^{1}$ Department of Midwifery, College of Medicine and Health Science, Wolkite University, Wolkite, Ethiopia \\ ${ }^{2}$ Department of Nursing, College of Medicine and Health Science, Wolkite University, Wolkite, Ethiopia \\ Correspondence should be addressed to Yibeltal Mesfin; hazardkuku@gmail.com
}

Received 11 June 2021; Accepted 1 August 2021; Published 16 August 2021

Academic Editor: Faustino R. Perez-Lopez

Copyright (c) 2021 Yibeltal Mesfin et al. This is an open access article distributed under the Creative Commons Attribution License, which permits unrestricted use, distribution, and reproduction in any medium, provided the original work is properly cited.

\begin{abstract}
Introduction. A dual contraceptive method is the usage of any modern contraceptive method with male or female condoms which could lower sexually transmitted diseases and unwanted pregnancy. Ethiopian standard utilization of dual contraceptive is low. The hassle is more severe for HIV/AIDS-infected people. Therefore, this review was aimed at assessing dual contraceptive utilization and factor associated with people living with HIV/AIDS in Ethiopia. Method. International databases (PubMed/MEDLINE, Hinari, Embase, African Journals Online, Scopus, and Google Scholar) and Ethiopian university repository online have been covered in this review. Microsoft Excel was used for extraction, and the Stata 14 software program was used for analysis. We detected the heterogeneity between studies using the Cochran $Q$ statistic and $I^{2}$ test. Publication bias was assessed by funnel plot and Egger's and Begg's tests. Result. The overall prevalence of dual contraceptive use among people living with HIV/AIDS was 27.73\% (95\% CI: 20.26-35.19) in Ethiopia. Discussion with the partner (OR: 3.78, 95\% CI: 3.08-4.69), HIV status disclosure to the spouse/partner (OR: 2.810, 95\% CI: 2.26-3.48), postdiagnosis counseling (OR: 5.00, 95\% CI: 3.71-6.75), schooling in secondary and above education (OR: 3.78, 95\% CI: 2.41-5.93), partner involvement in counseling (OR: 2.76, 95\% CI: 1.99-3.82), urban residence (OR: 2.84, 95\% CI 2.03-3.94), and having no fertility desire (OR: 4.01, 95\% CI 2.91-5.57) were significantly associated with dual contraceptive use. Conclusion. Dual contraceptive utilization among people living with HIV/AIDS was found to be low in Ethiopia. This will be a significant concern unless future intervention focuses on rural residence, involvement of the partner in postdiagnosis counseling, encouraging the people living with HIV/AIDS to disclose HIV status, and discussion with the partner. Providing counseling during the antenatal and postnatal period also enhances dual contraceptive use.
\end{abstract}

\section{Background}

Human immunodeficiency virus (HIV) is one of the significant pandemics the world faced and ongoing for 40 years [1]. According to the World Health Organization (WHO) 2020 report, about 37.6 million individuals worldwide are infected with HIV, while 1.5 million people become infected for the first time; nevertheless, the reduction is not fast enough to meet the objective of 500,000 people by 2020 [2]. Around 690,000 people died from acquired immunodeficiency disease syndrome- (AIDS-) related illnesses in 2020.
Despite the fact that the global new HIV infection reduced by 16 percent across the countries, there were still 6200 new infections each week for young girls between age 15 and 24 groups [2]. In sub-Saharan Africa, four in five new infections are between age 15 and 19 groups [3].

Globally, because of poor dual contraceptive utilization and unsafe sexual practice, over 2 million HIV-infected women become pregnant every year. Women infected with HIV/AIDS have unplanned pregnancies due to lack of access to health service, poor education, sexual violence, or failure of the family planning method [4]. The practices of poor dual 
contraceptive not only lead to unplanned pregnancies but also increase epidemic of HIV/AIDS and other strains of sexually transmitted infections (STIs) [4-6]. Evidences also showed that more than half of unintended pregnancy occurred among adolescent mothers ending with unsafe abortion [7].

A dual contraceptive method is the utilization of any modern contraceptive method along with male or female condoms which can decrease sexually transmitted disease (STD) and unwanted pregnancy. Consistent and correct use of a condom alone is highly effective for preventing STIs and unwanted pregnancy. However, typical use of a male condom as a contraception method may cause $15 \%$ of unwanted pregnancy [8]. Other hormonal contraceptives like combined oral contraceptive, injectable, implants, and intrauterine device protect pregnancy more but do not protect against HIV/AIDS and STIs $[8,9]$.

Utilization of dual contraceptive among people living with HIV/AIDS (PLWHA) is low in many developing countries, including Ethiopia. Even though a national report was not conducted, studies in various regional states of Ethiopia showed that dual contraceptive utilization among PLWHA is low. For example, in studies from Borena District, Northeast Ethiopia (19.4\%), Gondar (13.2\%), and Tigray (15.7\%), PLWHA used dual contraceptive methods [10-12].

Previous studies revealed that there are different enabling factors for dual contraceptive utilization for PLWHA. Urban residence, disclosing HIV status to a partner, secondary and higher educational level, partner involvement in counseling, postdiagnosis counseling about family planning, age of the women, discussion with the husband/partner, and having a higher CD4 cell count were significant factors affecting the use of dual contraceptive methods [11-13].

Despite the fact that there are publications on the prevalence of dual contraceptive usage and factors associated with PLWHA in Ethiopia's several regional states, there were no studies conducted that indicate the overall level of dual contraceptive use among Ethiopian PLWHA nationally. Therefore, the aim of this review is to have a summary of pooled prevalence dual contraceptive use and factor associated with PLWHA in Ethiopia.

\section{Methods}

2.1. Study Design and Search Strategy. We searched studies which reported on the prevalence of dual contraceptive use and associated factor with PLWHA in Ethiopia. This systematic review included studies found in PubMed/MEDLINE, Hinari, Embase, African Journals Online, Scopus, and Google Scholar and Ethiopian university repository online. All published articles as of May 10, 2021, were included in the review. A search of the reference list of the already identified studies to retrieve additional articles was done. The search terms used were "PLWHA dual contraceptive use OR HIVpositive women contraceptive use during ART OR determinants dual contraceptive of use AND Ethiopia". This was a systematic review and meta-analysis strictly adherent with the Preferred Reporting Items for Systematic Reviews and Meta-Analyses (PRISMA) protocol.

\subsection{Eligibility Criteria}

2.2.1. Inclusion Criteria. All cross-sectional (CS) published and unpublished studies which reported the prevalence and associated factor with PLWHA in Ethiopia with English language research articles were included in the review. Studies were searched from April 1 to 30, 2021.

2.2.2. Exclusion Criteria. Articles whose full text and abstract were unable to be accessed were excluded. Duplicated studies were also checked and removed before the pooled process started.

2.3. Data Extraction and Quality Assessment. All articles were extracted and reviewed by two authors (MA and SG) independently with the standard Microsoft Excel spreadsheet form. Disagreement between these authors is handled by discussion with other two authors (YM and BT). The sample size, year of publication, study design, name of authors, region where the study was conducted, prevalence of $95 \%$ $\mathrm{CI}$, response rate, and odds ratio were extracted from the original studies.

2.3.1. Outcome Variable. The primary outcome variable of this systematic review and meta-analysis was the prevalence of dual contraceptive use among PLWHA. This was calculated by the pooled prevalence of dual contraceptive use among PLWHA.

The other outcome variable of this review was factors associated with dual contraceptive use among PLWHA in any age group. Calculated by factors associated with dual contraceptive use to each study log, an odds ratio was used.

(1) Dual contraceptive use: dual contraceptive use is the utilization of a condom plus any other family planning method by PLWHA for the previous one month

(2) Modern methods: the modern methods include sterilization, pills, intrauterine device, injectable, implants, and condom

(3) Natural methods: the natural methods include temperature awareness, calendar, cervical mucus, lactational amenorrhoea, and coitus interruptus

2.3.2. Data Processing and Analysis. The Microsoft Excel spreadsheet form was used for extraction, and the Stata 14 software program was used for analysis. The overall pooled prevalence of dual contraceptive use was computed using random effects meta-analysis. Publication bias was assessed by funnel plot through visual assessment. Egger's and Begg's tests at $5 \%$ were also performed to detect publication bias. We detected the heterogeneity between studies using the Cochran $Q$ statistic and $I^{2}$ test. Subgroup analysis by region and year was also calculated to compare dual contraceptive utilization across regions of the country. The point prevalence with a $95 \%$ confidence interval (CI) was presented with forest plot format. The relation between the dual contraceptive and the associated factors was determined by the adjusted odds ratio (AOR). 


\section{Result}

3.1. Description of the Included Study. We retrieved 145 studies from the PubMed/MEDLINE, Hinari, Embase, African Journals Online, Scopus, and Google Scholar and Ethiopian university repository online research articles. After the duplicated studies were removed, 81 articles remained. Then, 70 articles were excluded through the reading of their abstract and titles. Thus, 12 full-text articles were assessed for eligibility criteria, further yielding the exclusion of 1 article. Finally, the remaining 11 articles were used to assess the pooled prevalence of dual contraceptive use and its determinant (Figure 1).

3.2. Characteristics of Identified Studies. In this review, 11 cross-sectional studies were included. All studies were conducted after 2014. About 4386 study population was included to estimate the pooled prevalence. Five regions were represented for this review. Three from the Amhara region, three from the Oromia region, two from the Southern Nations, Nationalities, and Peoples' Region (SNNPR), two from the Tigray region, and one study from the Benishangul-Gumuz region in Ethiopia were included (Table 1).

3.3. Prevalence of Dual Contraceptive Utilization. The lowest prevalence of dual contraceptive utilization was $13 \%$ observed in a study conducted in the Amhara region, and the highest prevalence was also $53.4 \%$ observed in a study conducted in the Amhara region. The pooled national level of dual contraceptive utilization was $27.73 \%$ (95\% CI: $20.26 \%, 35.19 \%$ ) based on the random effects analysis (Figure 2).

3.4. Subgroup Analysis. Subgroup analysis by region and year was also calculated to compare dual contraceptive utilization across regions of the country. Accordingly, the highest dual contraceptive utilization was observed in BenishangulGumuz $(40.94 \%$, 95\% CI: 36.14, 45.74) followed by the Oromia region $(32.27 \%, 95 \%$ CI: 12.97, 51.58) and Tigray region (30.09\%, 95\% CI: 1.28, 58.91), whereas the lowest utilization of dual contraceptive $(20.26 \%, 95 \%$ CI: $10.99,29.53)$ was observed in the Amhara region (Figure 3).

3.5. Heterogeneity and Publication Bias. The $I^{2}$ (variation in ES attributable to heterogeneity) test result showed that there was high heterogeneity with $I^{2}=97.2$, at $p$ value $<0.0001$. We also assessed the presence of publication bias among studies included in the review. The funnel plot showed that there was an asymmetrical distribution of the included studies through visual inspection, which indicates that there was potential publication bias (Figure 4). However, the Egger regression test $(p=0.032)$ and Begg rank correlation statistics $(p=0.161)$ showed that there was no evidence of potential publication bias.

3.6. Sensitivity Analysis. We conducted a sensitivity analysis using a random effects model. Hence, the results showed that no single study influenced the overall pooled proportion of dual contraceptive utilization among PLWHA (Figure 5).

3.7. Factors Associated with Dual Contraceptive Utilization. The current review identified different factors associated with dual contraceptive utilization for PLWHA in Ethiopia. Sig- nificantly associated factors were residence, disclosure, discussion with the partner about family planning, counseling about family planning, positive partner, fertility desire, and educational status.

The PLWHA residence was significantly associated with dual contraceptive. Using the studies included in the group of meta-analysis, PLWHA who live in urban areas were 2.84 times more likely to use dual contraceptive (AOR: $2.84,95 \%$ CI $2.03,3.94$ ) than those living in rural residence.

In this review, PLWHA who have discussed with their partner were 3.78 times more likely to use contraceptives (AOR: 3.78, 95\% CI: 3.08-4.69) than those who have not discussed with their partner.

Other significant factors associated with dual contraceptive utilization were disclosure of HIV status with the partner. Dual contraceptive use was more likely on PLWHA who disclose their HIV status to their partner than on their counterpart (AOR: 2.810, 95\% CI: 2.26-3.48).

Those PLWHA who counseled about family planning ever were 5 times more likely to use dual contraceptives as compared with those not getting any family planning counseling (AOR: 5.00, 95\% CI: 3.71-6.75). Similarly, individuals whose partner is involved in counseling were 2.76 times more likely to utilize dual contraceptives as compared with their counterpart (AOR: 2.76, 95\% CI: 1.99-3.82).

Educational status is also a novel factor affecting dual contraceptive use. PLWHA who finished secondary and above education were 3.78 times more likely to use dual contraceptives than their counterpart (AOR: 3.78, 95\% CI: 2.415.93). Additionally, PLWHA who have no desire were 4.01 times more likely to use dual contraceptive than those having the desire in the future ( $\mathrm{OR}=4.01,95 \%$ CI 2.91, 5.57).

\section{Discussion}

Dual contraceptive uses among PLWHA have an enormous benefit to controlling unwanted pregnancies, decreasing unsafe abortions, and reducing HIV-positive deliveries. So this review was aimed at assessing dual contraceptive utilization and factor associated with people living with HIV/AIDS in Ethiopia. In this meta-analysis, the pooled prevalence of dual contraceptive use among PLWHA in Ethiopia was $27.73 \%$ (95\% CI: 20.26\%, 35.19\%). Despite the fact that no comparable meta-analysis study on this specific topic has been conducted in the area, the overall prevalence of dual contraceptive use by PLWHA is analogous to studies conducted in southeast Nigeria (27.7\%) [14]; Soweto, South Africa (33\%) [15]; Brazil (28\%) [16]; and India (23\%) [17]. This finding is higher than studies conducted in Uganda (3.5\%) [18] and Zambia (17.7\%) [19]. But it is lower than studies conducted in Bungoma County, Kenya (38\%.5) [20]; Nigeria (45\%) [21]; and United States of America (47\%) [22]. This difference might be because of regional variation and variation in guidelines of reproductive health and health policy of the country.

This study showed that HIV-positive women who were living in urban areas were more likely to use dual contraceptive methods as compared to those living in rural areas. This is consistent with the finding from Malawi and Togo [23, 24]. 


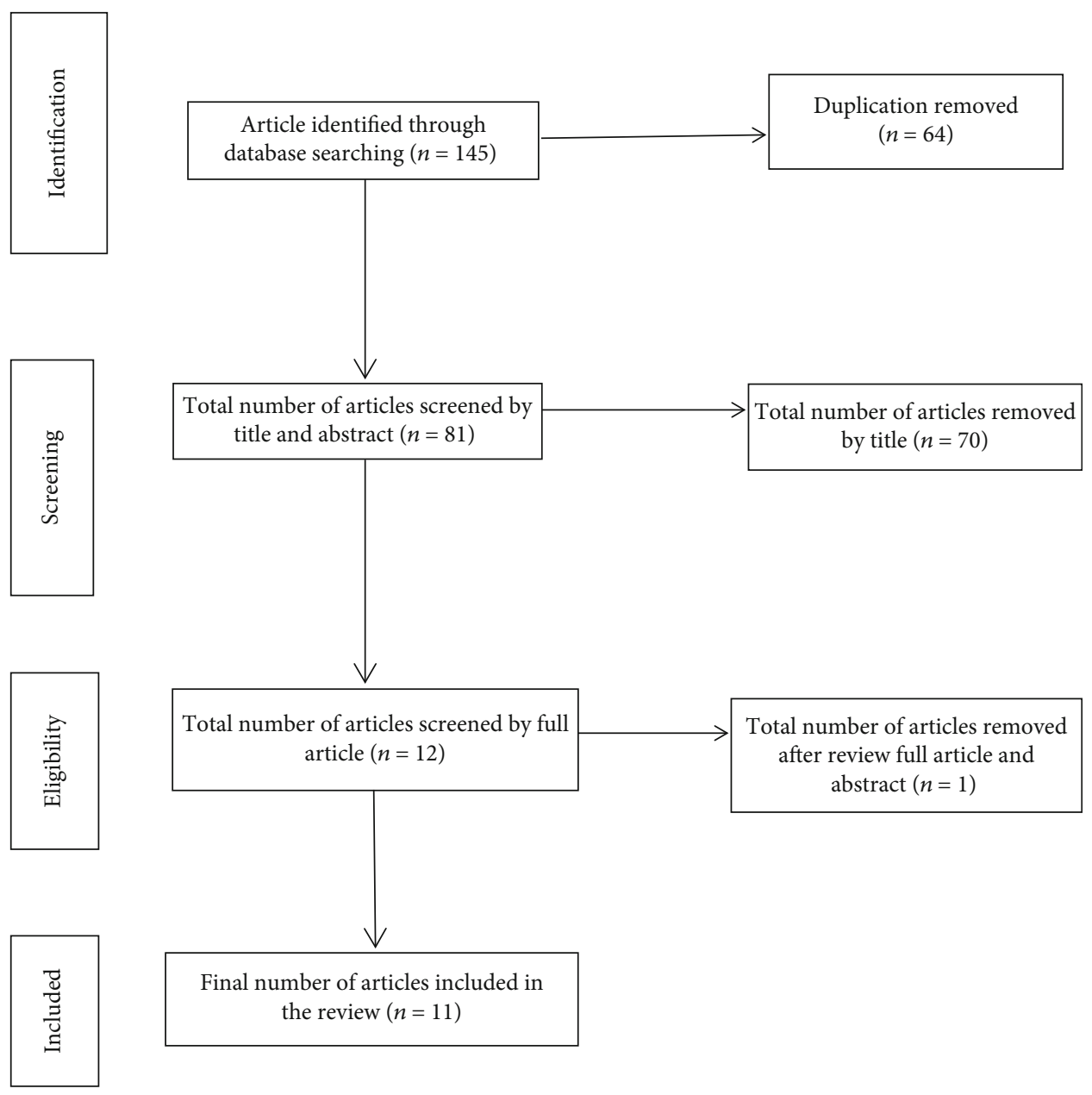

Figure 1: Flow diagram of study selection for systematic and meta-analysis of dual contraceptive use in Ethiopia.

TABle 1: Descriptive summary of 11 studies included in the meta-analysis of dual contraceptive use in Ethiopia.

\begin{tabular}{|c|c|c|c|c|c|}
\hline Name of author & Publication year & Region & Study design & Sample size & Response rate \\
\hline Assefa Alemayehu & 2018 & Benishangul-Gumuz & CS & 403 & 100 \\
\hline Demissie et al. & 2015 & Oromia & CS & 357 & 95.2 \\
\hline Gebrehiwot et al. & 2017 & Tigray & CS & 331 & 97.9 \\
\hline Abay et al. & 2020 & Amhara & CS & 563 & 0.98 \\
\hline Dereje Beyissia & 2019 & Oromia & CS & 339 & 94.1 \\
\hline Halefom Kalayu & 2017 & Tigray & CS & 520 & 99.2 \\
\hline Amare et al. & 2021 & Amhara & CS & 417 & 98.8 \\
\hline Tilahun Beyene & 2019 & SNNPR & CS & 269 & 95.9 \\
\hline Erashi et al. & 2015 & SNNPR & CS & 246 & 98.78 \\
\hline Reta et al. & 2019 & Amhara & CS & 630 & 98.25 \\
\hline Tilahun et al. & 2021 & Oromia & CS & 311 & 97.7 \\
\hline
\end{tabular}

The explanation for this might be because of the variation in accessing information and services related to this area. The urban women more easily accessed important information and services related to dual contraceptive utilization through different sources like social media and also from their daily social interpersonal relationships.

In this review, PLWHA who have discussed with their partner were 3.78 times more likely to use dual contraceptives 


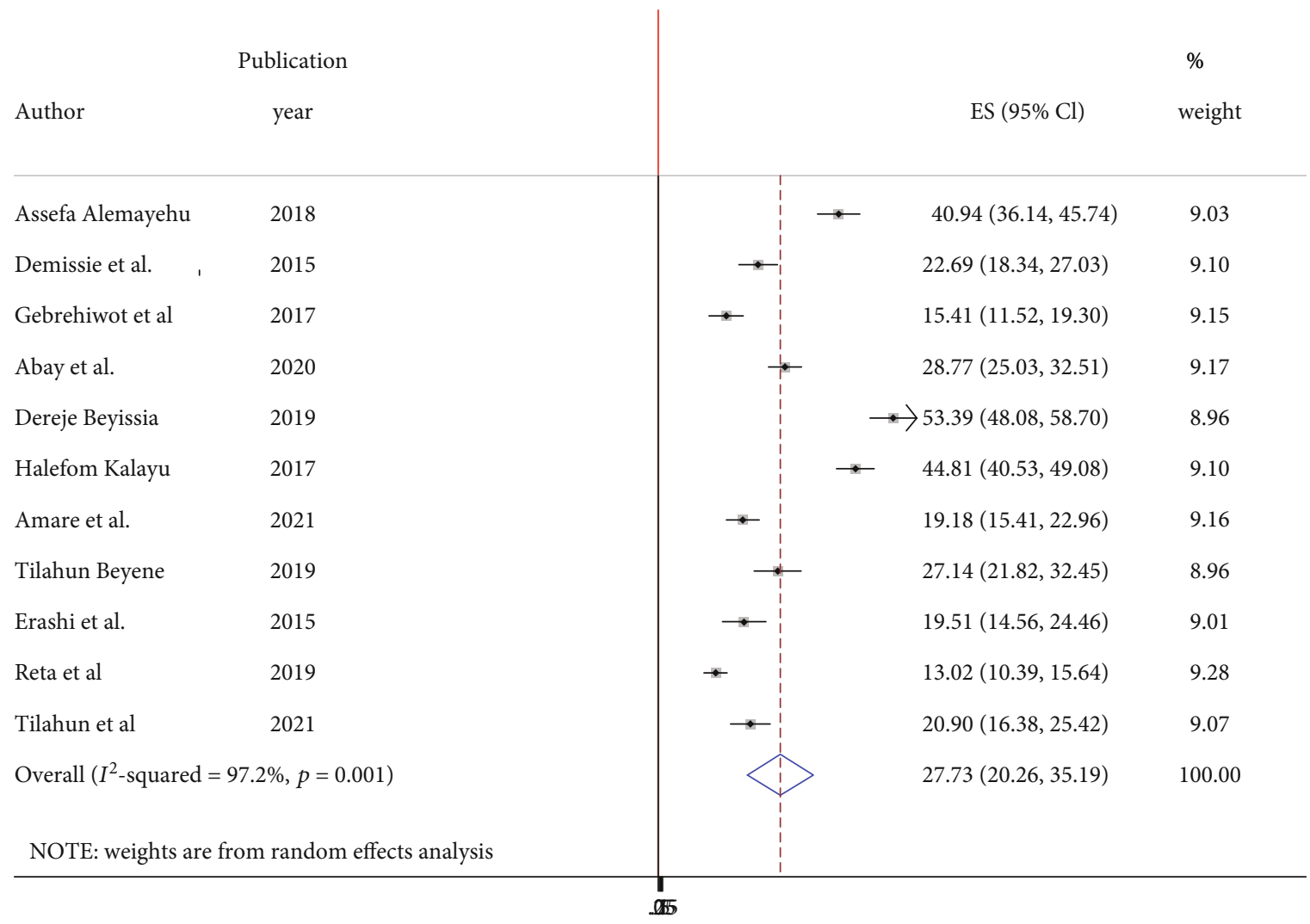

FIGURE 2: Forest plot in pooled prevalence of dual contraceptive use in Ethiopia.

(AOR: 3.78, 95\% CI: 3.08-4.69) than those who have not discussed with their partner. This was supported by studies done in Tanzania, Zambia, and Malawi $[17,25,26]$. This is clear that an open communication between couples about sexual and reproductive issue helps to improve contraceptive use. It implies that the need to introduce open discussion between couples on sexual and reproductive issue helps not only to increase dual contraceptive use but also to improve adherence on ART and PMTCT and minimize loss of follow-up for PLWHA [27].

This meta-analysis showed that PLWHA who disclose their HIV statuses to their spouse/partner were found to have significantly increased utilization of dual contraceptives. This finding was supported by studies done in Kenya, Zimbabwe, and Ghana $[20,28,29]$. It is because disclosure facilitating open discussion between partners helps them to mutually accept the importance of dual contraceptive use.

Educational status was also a novel factor affecting dual contraceptive use. People living with HIV/AIDS who finished secondary and above education were 3.78 times more likely to use dual contraceptives than their counterpart. Similar report supports this systematic review from Brazil and Uganda [30, 31]. This can be explained by the fact that educated women have more awareness of dual contraceptive on prevention of HIV/AIDS, unwanted pregnancy, and sexually transmitted disease. Therefore, educating PLWHA is a prominent factor to increase dual contraception use.
PLWHA who had no fertility desire were more likely to use dual contraceptive as compared to their counterpart. This finding is in line with studies from South Africa and Kenya $[15,32]$. This may be because couples living with HIV/AIDS who did not want to have a child in the future were more likely use dual contraceptive to protect unwanted pregnancy and STI transmission.

This meta-analysis indicated that PLWHA who received postdiagnosis counseling on family planning were more likely to utilize dual contraceptive methods. The finding is supported by studies from India and Tanzania [17, 25]. This might be explained by the fact that PLWHA have been counseled during their follow-up by a healthcare provider to get more knowledge about reproductive health. Having adequate knowledge about family planning leads to the use of more dual contraceptive to minimize HIV transmission and unintended pregnancy.

Furthermore, the meta-analysis identified that dual contraceptive use was higher in partner involvement in counseling about family planning. The finding was supported by a study from India [17]. It is found in this finding that involvement of the partner in counseling helps the couple for open discussion for their sexual and reproductive health service utilization.

Finally, this systematic review and meta-analysis has limitations. All studies included in this study were cross-sectional, and establishing a cause and effect is imposable due to 


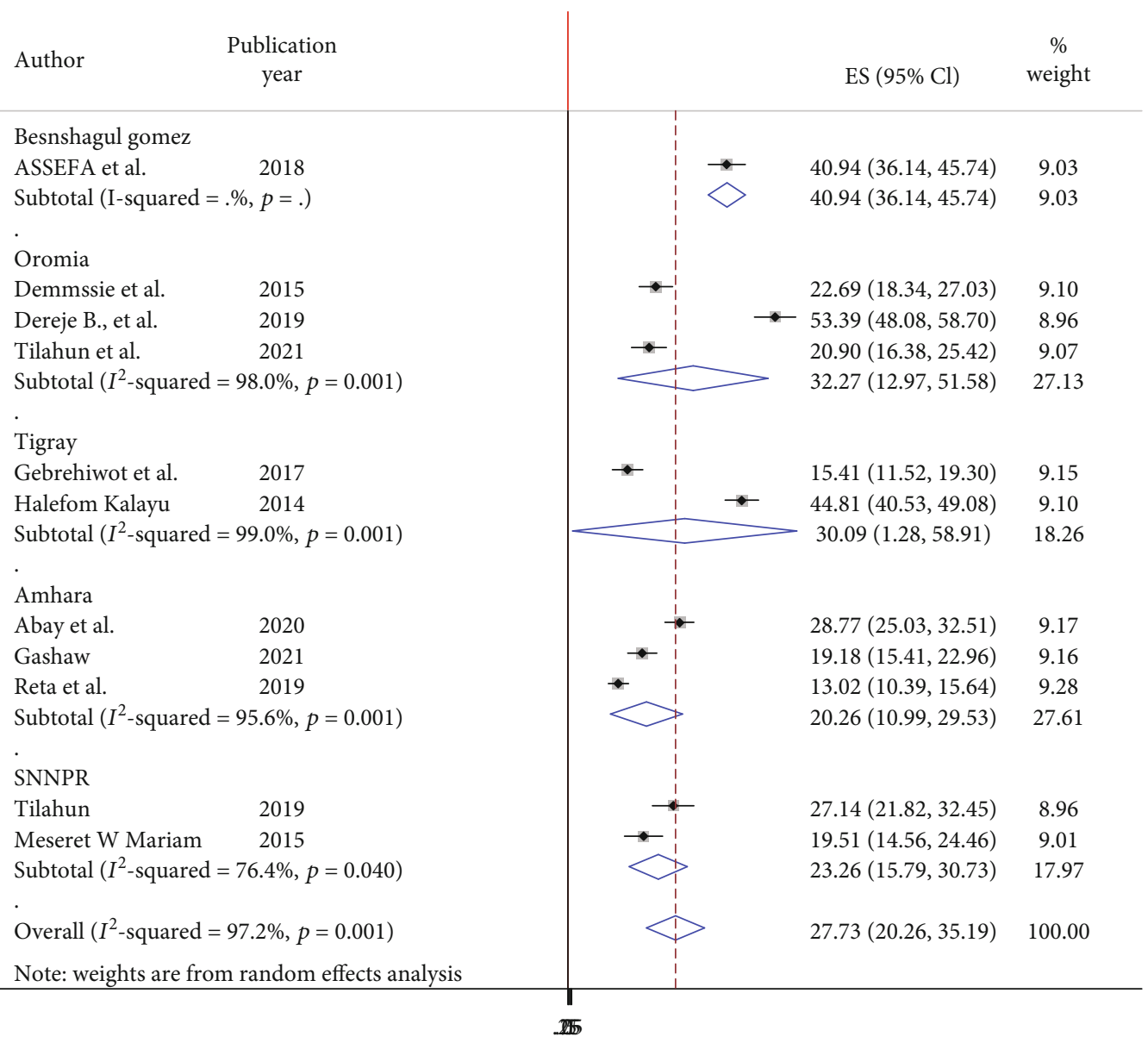

FIGURE 3: Forest plot showing the subgroup analysis of dual contraceptive in Ethiopia by region.

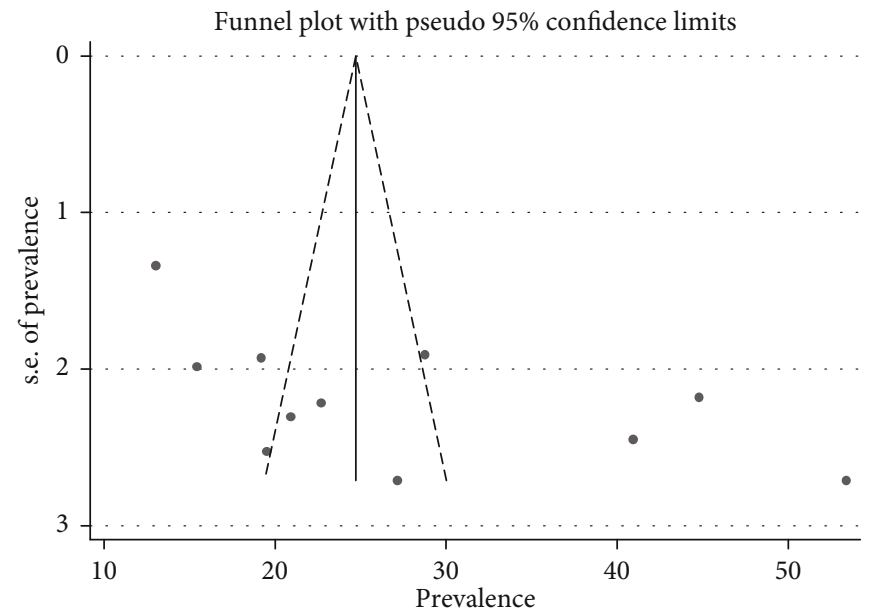

Figure 4: Funnel plot depicting publication bias of studies reporting the dual contraceptive use in Ethiopia. 


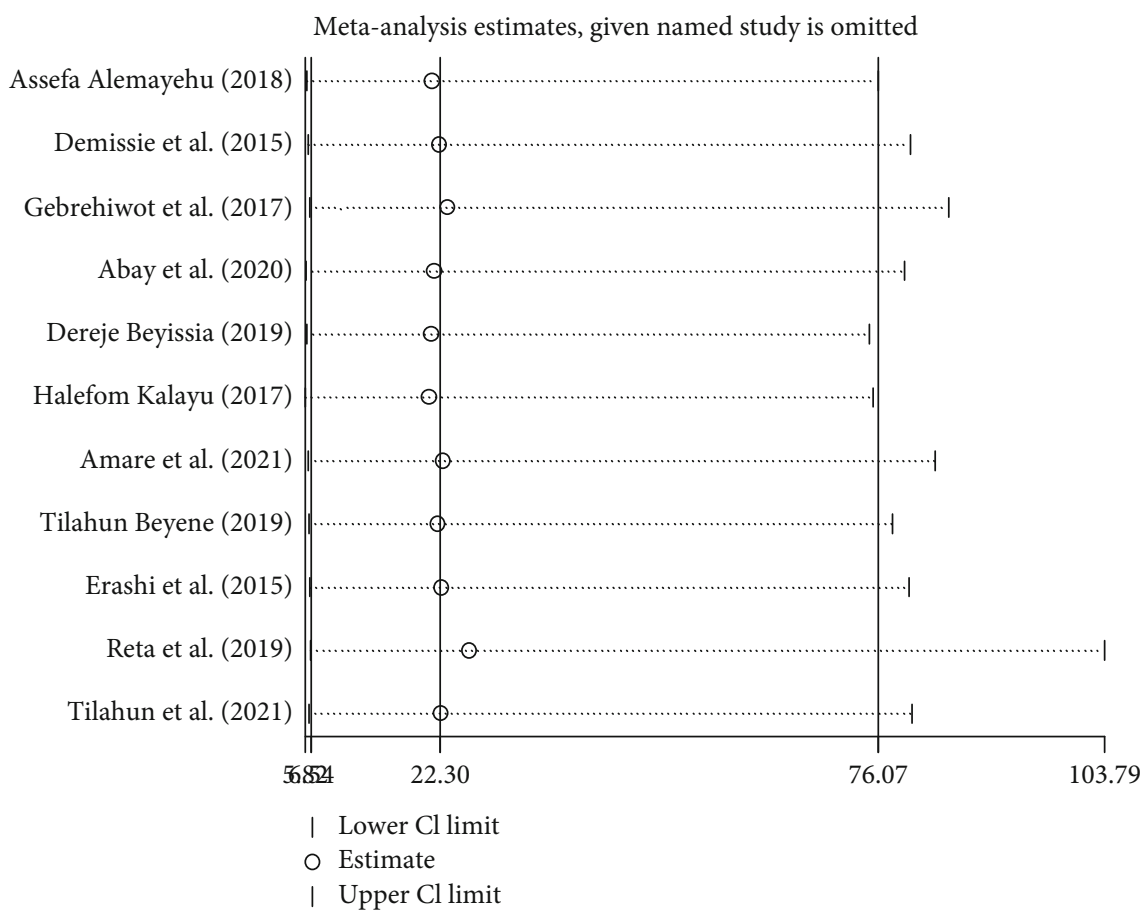

FIGURE 5: Sensitivity analysis of the prevalence of dual contraceptive use in Ethiopia.

study design limitation. Additionally, the studies were from five regions only which may restrict the representativeness of the finding.

\section{Conclusion}

The overall utilization of the dual contraceptive method is low. Urban residence, disclosure of HIV status, discussion with the partner about family planning, counseling about family planning, partner involvement in counseling, fertility desire, and educational status were factors affecting utilization of dual contraceptive for PLWHA. Therefore, based on the study findings, husband involvement and providing counseling about contraceptive methods for PLWHA should be encouraged, and there is a need for an intensified effort to improve reproductive health service utilization. Healthcare providers in charge of HIV caregiving must also integrate family planning service counseling in HIV care during follow-up visits.

\section{Abbreviations}

AOR: $\quad$ Adjusted odds ratio

AIDS: Acquired immunodeficiency disease syndrome

CS: Cross-sectional

CI: $\quad$ Confidence interval

OR: $\quad$ Odds ratio

HIV: $\quad$ Human immunodeficiency virus

PLWHA: People living with HIV/AIDS

SNNPR: Southern Nations, Nationalities, and Peoples' Region

STIs: $\quad$ Sexually transmitted infections

WHO: World Health Organization.

\section{Data Availability}

Full datasets and other materials about this study could be obtained from the corresponding author upon reasonable request.

\section{Conflicts of Interest}

The authors declare that there is no financial and nonfinancial conflict of interest regarding the publication of this paper.

\section{Authors' Contributions}

All authors contributed to data analysis and drafting or revising the article, gave the final approval of the version to be published, and agreed to be accountable for all of the work.

\section{References}

[1] B. X. Tran, H. T. Phan, C. A. Latkin et al., "Understanding global HIV stigma and discrimination: are contextual factors sufficiently studied? (GAPRESEARCH)," International journal of environmental research and public health, vol. 16, no. 11, p. 1899, 2019.

[2] Global HIV Statistics, UNAIDS, 2021, https://www.unaids.org/ sites/default/files/media_asset/UNAIDS_FactSheet_en.pdf.

[3] A. B. Kharsany and Q. A. Karim, "HIV infection and AIDS in sub-Saharan Africa: current status, challenges and opportunities," The open AIDS journal, vol. 10, no. 1, pp. 34-48, 2016.

[4] D. Josefson, "Unsafe sexual practices are common, say US studies," BMJ, vol. 327, no. 7405, pp. 10-d-10-0, 2003.

[5] J. R. Willard Cates and M. J. Steiner, "Dual protection against unintended pregnancy and sexually transmitted infections: 
what is the best contraceptive approach?," Sexually transmitted diseases, vol. 29, no. 3, pp. 168-174, 2002.

[6] M. C. SI, R. Buzdugan, L. J. Ralph et al., "Unmet need for family planning, contraceptive failure, and unintended pregnancy among HIV-infected and HIV-uninfected women in Zimbabwe," PLoS One, vol. 9, no. 8, article e105320, 2014.

[7] A. Singh, A. Singh, and B. Mahapatra, "The consequences of unintended pregnancy for maternal and child health in rural India: evidence from prospective data," Maternal and child health journal, vol. 17, no. 3, pp. 493-500, 2013.

[8] J. Trussell and K. Guthrie, "Choosing a contraceptive: efficacy, safety, and personal considerations," in Contraceptive Technology, R. A. Hatcher, J. Trussell, A. L. Nelson, W. Cates, F. H. Stewart, and D. Kowal, Eds., pp. 19-47, Ardent Media, Inc, New York (NY), 19th edition, 2007.

[9] W. CATES, M. STEINER, and E. Raymond, "Dual vs. duel (ing) protection against unintended pregnancy and sexually transmitted infections: what is that best contraceptive approach? Research Triangle Park," in USAID Open Forum on Condom Promotion and Dual Protection, Family Health International, Washington, DC, 2000.

[10] N. C. Gashaw Amare and A. M. Mekonen, "Dual contraceptive use and associated factors among reproductive age group on antiretroviral therapy in Borena District, Northeast Ethiopia: a cross-sectional study," HIVIAIDS (Auckland, NZ), vol. 13, p. 107, 2021.

[11] M. M. Reta, G. A. Tessema, and G. Shiferaw, "Prevalence of dual contraceptive use and associated factors among HIV positive women at University of Gondar Hospital, Northwest Ethiopia," BMC research notes, vol. 12, no. 1, pp. 1-7, 2019.

[12] S. W. Gebrehiwot, G. A. Azeze, C. C. Robles, and Y. M. Adinew, "Utilization of dual contraception method among reproductive age women on antiretroviral therapy in selected public hospitals of Northern Ethiopia," Reproductive health, vol. 14, no. 1, pp. 1-9, 2017.

[13] M. W. Mariam, F. Yadassa, and T. Tema, "Dual-contraceptive method utilization and associated factors among HIV positive women attending art clinic in Gebretsadik Shawo Hospital, SNNPR, South West Ethiopia," Women's Health Care, vol. 4, no. 6, 2015.

[14] L. O. Lawani, A. K. Onyebuchi, and C. A. Iyoke, "Dual method use for protection of pregnancy and disease prevention among HIV-infected women in South East Nigeria," BMC women's health, vol. 14, no. 1, p. 39, 2014.

[15] A. Kaida, F. Laher, S. A. Strathdee et al., "Contraceptive use and method preference among women in Soweto, South Africa: the influence of expanding access to HIV care and treatment services," PLoS One, vol. 5, no. 11, article e13868, 2010.

[16] J. Magalhães, E. Amaral, P. C. Giraldo, and J. A. Simoes, "HIV infection in women: impact on contraception," Contraception, vol. 66, no. 2, pp. 87-91, 2002.

[17] V. Chakrapani, T. Kershaw, M. Shunmugam, P. A. Newman, D. H. Cornman, and R. Dubrow, "Prevalence of and barriers to dual-contraceptive methods use among married men and women living with HIV in India," Infectious diseases in obstetrics and gynecology, vol. 2011, Article ID 376432, 8 pages, 2011.

[18] J. Heys, W. Kipp, G. S. Jhangri, A. Alibhai, and T. Rubaale, "Fertility desires and infection with the HIV: results from a survey in rural Uganda," AIDS (London, England), vol. 23, Suppl 1, pp. S37-S45, 2009.
[19] C. J. Chibwesha, M. S. Li, C. K. Matoba et al., "Modern contraceptive and dual method use among HIV-infected women in Lusaka, Zambia," Infectious diseases in obstetrics and gynecology, vol. 2011, 8 pages, 2011.

[20] A. M. Mulongo, R. W. Lihana, J. Githuku, Z. Gura, and S. Karanja, "Factors associated with uptake of dual contraception among HIV-infected women in Bungoma County, Kenya: a cross-sectional study," The Pan African Medical Journal, vol. 28, Suppl 1, p. 2, 2017.

[21] L. Adeokun, J. E. Mantell, E. Weiss et al., "Promoting dual protection in family planning clinics in Ibadan, Nigeria," International Family Planning Perspectives, vol. 28, no. 2, pp. 87-95, 2002.

[22] T. E. Wilson, L. J. Koenig, E. Walter, I. Fernandez, and K. Ethier, "Dual contraceptive method use for pregnancy and disease prevention among HIV-infected and HIV-uninfected women: the importance of an event-level focus for promoting safer sexual behaviors," Sexually transmitted diseases, vol. 30, no. 11, pp. 809-812, 2003.

[23] D. Habte and J. Namasasu, "Family planning use among women living with HIV: knowing HIV positive status helpsresults from a national survey," Reproductive health, vol. 12, no. 1, pp. 1-11, 2015.

[24] I. Yaya, A. A. Patassi, D. E. Landoh et al., "Modern contraceptive use among HIV-infected women attending HIV care centres in Togo: a cross-sectional study," BMJ Open, vol. 8, no. 4, article e019006, 2018.

[25] D. J. Damian, J. M. George, E. Martin, B. Temba, and S. E. Msuya, "Prevalence and factors influencing modern contraceptive use among HIV-positive women in Kilimanjaro region, northern Tanzania," Contraception and Reproductive Medicine, vol. 3, no. 1, p. 7, 2018.

[26] K. Grabbe, R. Stephenson, B. Vwalika et al., "Knowledge, use, and concerns about contraceptive methods among serodiscordant couples in Rwanda and Zambia," Journal of women's health, vol. 18, no. 9, pp. 1449-1456, 2009.

[27] S. E. Msuya, E. Mbizvo, A. Hussain, J. Uriyo, N. Sam, and B. Stray-Pedersen, "Low male partner participation in antenatal HIV counselling and testing in northern Tanzania: implications for preventive programs," AIDS Care, vol. 20, no. 6, pp. 700 709, 2008.

[28] T. Magwali, M. Steiner, H. Toms, and J. Brown, How are condoms used in a family planning setting: evidence from Zimbabwe, 2005.

[29] R. K. Wanyenze, J. K. B. Matovu, M. R. Kamya, N. M. Tumwesigye, M. Nannyonga, and G. J. Wagner, "Fertility desires and unmet need for family planning among HIV infected individuals in two HIV clinics with differing models of family planning service delivery," BMC Women's Health, vol. 15, no. 1, p. 5, 2015.

[30] K. Tsuyuki, R. M. Barbosa, and A. de Araujo Pinho, "Dual protection and dual methods in women living with HIV: the Brazilian context," Journal of sexually transmitted diseases, vol. 2013, 8 pages, 2013.

[31] J. Beyeza-Kashesya, F. Kaharuza, A. M. Ekström, S. Neema, A. Kulane, and F. Mirembe, "To use or not to use a condom: a prospective cohort study comparing contraceptive practices among HIV-infected and HIV-negative youth in Uganda," BMC infectious diseases, vol. 11, no. 1, pp. 1-11, 2011.

[32] G. Antelman, A. Medley, R. Mbatia et al., "Pregnancy desire and dual method contraceptive use among people living with HIV attending clinical care in Kenya, Namibia and Tanzania," Journal of Family Planning and Reproductive Health Care, vol. 41, no. 1, p. e1, 2015. 